Bundesgesundheitsbl 2018 $\cdot 61: 420-431$ https://doi.org/10.1007/s00103-018-2712-4 Online publiziert: 27. Februar 2018 (c) Der/die Autor(en) 2018. Dieser Artikel ist eine Open-Access-Publikation.

CrossMark

Wolfram J. Herrmann ${ }^{1,6} \cdot$ Cornelia Weikert $^{2,7} \cdot$ Manuela Bergmann $^{3} \cdot$

Heiner Boeing ${ }^{3}$. Verena A. Katzke ${ }^{8} \cdot$ Rudolf Kaaks $^{9} \cdot$ Daniel Tiller ${ }^{16}$.

Karin Halina Greiser ${ }^{10,16} \cdot$ Margit Heier $^{9,10}$. Christa Meisinger ${ }^{10,11,20}$.

Carsten Oliver Schmidt ${ }^{12} \cdot$ Hannelore Neuhauser ${ }^{7,13}$. Christin Heidemann ${ }^{13}$. Claus Jünger ${ }^{14,18} \cdot$ Philipp S. Wild ${ }^{14,17,18,19} \cdot$ Sara Helena Schramm ${ }^{15} \cdot$ Karl- $^{15}$

Heinz Jöckel ${ }^{15}$ - Marcus Dörr ${ }^{4,5} \cdot$ Tobias Pischon $^{1,6,7}$

${ }^{1}$ Forschergruppe Molekulare Epidemiologie, Max-Delbrück-Centrum für Molekulare Medizin in der Helmholtz-Gemeinschaft (MDC), Berlin, Deutschland; ${ }^{2}$ Fachgruppe Risiken besonderer Bevölkerungsgruppen und Humanstudien, Abteilung Lebensmittelsicherheit, Bundesinstitut für Risikobewertung, Berlin, Deutschland; ${ }^{3}$ Deutsches Institut für Ernährungsforschung Potsdam-Rehbrücke, Nuthetal, Deutschland; ${ }^{4}$ Klinik und Poliklinik für Innere Medizin B, Universitätsmedizin Greifswald,

Greifswald, Deutschland; ${ }^{5}$ Partnerstandort Greifswald, Deutsches Zentrum für Herz-Kreislauf-Forschung, Greifswald, Deutschland; ${ }^{6}$ Charité - Universitätsmedizin Berlin, Berlin, Deutschland; ${ }^{7}$ Partnerstandort Berlin, Deutsches Zentrum für Herz-Kreislauf-Forschung, Berlin, Deutschland; ${ }^{8}$ Abteilung Epidemiologie von Krebserkrankungen, Deutsches Krebsforschungszentrum (DKFZ), Heidelberg, Deutschland; ${ }^{9}$ MONICA/KORA Herzinfarktregister, Klinikum Augsburg, Augsburg, Deutschland; ${ }^{10}$ Institut für Epidemiologie II, Helmholtz Zentrum München, Deutsches Forschungszentrum für Gesundheit und Umwelt (GmbH), Neuherberg, München, Deutschland; "Lehrstuhl für Epidemiologie, LudwigMaximilians-Universität München, München, Deutschland; ${ }^{12}$ Institut für Community Medicine, Universitätsmedizin Greifswald, Greifswald, Deutschland; ${ }^{13}$ Abteilung für Epidemiologie und Gesundheitsmonitoring, Robert Koch-Institut, Berlin, Deutschland; ${ }^{14}$ Präventive Kardiologie und Medizinische Prävention, Zentrum für Kardiologie, Universitätsmedizin Mainz, Mainz, Deutschland; ${ }^{15}$ Institut für Medizinische Informatik, Biometrie und Epidemiologie, Universitätsklinikum Essen, Essen, Deutschland; ${ }^{16}$ Institut für Medizinische Epidemiologie, Biometrie und Informatik (IMEBI), Martin-LutherUniversität Halle-Wittenberg, Halle (Saale), Deutschland; ${ }^{17}$ Zentrum für Thrombose und Hämostase, Universitätsmedizin Mainz, Mainz, Deutschland; ${ }^{18}$ Standort Rhein-Main, Deutsches Zentrum für HerzKreislaufforschung (DZHK), Mainz, Deutschland; ${ }^{19}$ Zentrum für Translationale Vaskuläre Biologie (CTVB), Universitätsmedizin Mainz, Mainz, Deutschland; ${ }^{20}$ UNIKA-T Augsburg, Augsburg, Deutschland

\title{
Erfassung inzidenter kardiovaskulärer und metabolischer Erkrankungen in epidemiologischen Kohortenstudien in Deutschland
}

hortenstudien einen wichtigen Beitrag. Zentrale Aspekte bei der Durchführung dieser Studien sind neben der Erhebung möglicher Einflussfaktoren insbesondere die Erfassung und Klassifikation neu aufgetretener (inzidenter) Erkrankungen während der Nachbeobachtungszeit. Zur Erfassung und Validierung inzidenter kardiovaskulärer Erkrankungen gibt es in Deutschland bisher nur wenige regionale Register, auf die zurückgegriffen werden kann [3-5]. Erschwerend für eine Validierung inzidenter kardiovaskulärer und metabolischer Erkrankungen kommt eine relativ starke Fragmentie- rung des Gesundheitssystems hinzu: An der Versorgung von kranken Menschen sind meist viele verschiedene behandelnde Personen beteiligt und es liegen nicht - wie in anderen Ländern - alle Befunde bei einem festen Hausarzt vor. Die Erfassung und Validierung neu aufgetretener Erkrankungen in epidemiologischen Studien in Deutschland ist daher eine Herausforderung.

Gerade für die Planung der Nachbeobachtungsphase von Kohortenstudien in Deutschland, wie beispielsweise der NAKO-Gesundheitsstudie (Nationale Kohorte), sind Erkenntnisse aus be- 
reits länger bestehenden Studien wichtig [6]. Ziel der vorliegenden Arbeit war es daher, populationsbasierte Kohortenstudien in Deutschland mit bereits erreichten inzidenten kardiovaskulären und metabolischen Erkrankungen zusammenzutragen und anhand der Herangehensweisen in diesen Studien Herausforderungen, Schwierigkeiten und bestehende Lösungsansätze für die Erfassung und Klassifikation der Endpunkte Herzinfarkt, Diabetes mellitus Typ 2, Schlaganfall, arterielle Hypertonie und Herzinsuffizienz vorzustellen.

\section{Methodik}

Von der gemeinsamen Arbeitsgruppe „Epidemiologie der Herz-Kreislauf- und Stoffwechsel-Erkrankungen“ der Deutschen Gesellschaft für Epidemiologie (DGEpi), der Deutschen Gesellschaft für Medizinische Informatik, Biometrie und Epidemiologie (GMDS) und der Deutschen Gesellschaft für Sozialmedizin und Prävention (DGSMP) wurde in einem ersten Schritt eruiert, welche Kohortenstudien es gibt, die zur Zeit durchgeführt werden und in denen bereits inzidente kardiovaskuläre und metabolische Erkrankungen vorliegen. Mit vertretenden Personen dieser Studien wurde am 26.11.2015 am MaxDelbrück-Centrum für Molekulare Medizin in der Helmholtz-Gemeinschaft (MDC) ein Workshop mit dem Titel „Erhebung und Validierung von kardiovaskulären Ereignissen in epidemiologischen Studien - methodische und praktische Aspekte" durchgeführt. Dabei stellten diese ihre verschiedenen Vorgehensweisen und Methoden zur Identifizierung, Klassifizierung und Validierung der kardiovaskulären und metabolischen Endpunkte sowie die genutzten Datenquellen vor, woran sich eine Diskussion anschloss.

Die Beiträge und Diskussionspunkte sind im Nachgang für die Publikation systematisch ergänzt und aufbereitet worden. Dazu wurden an alle vertretenden Personen Erhebungsbögen zu ihren Studien verschickt und ausgewertet. Gefragt wurde insbesondere nach den validierten inzidenten Fällen (einschließlich letaler Fälle), nach den Datenquellen zur Er- fassung und Validierung inzidenter Fälle und zu dem Vorgehen der Erfassung und Validierung von Herzinfarkt, Diabetes mellitus Typ 2, Schlaganfall, Herzinsuffizienz und arterieller Hypertonie. Falls vorhanden, haben die Studien darüber hinaus Daten zum Anteil bestätigter und falsifizierter als potenzielle Erkrankungsfälle identifizierter Personen geliefert und Angaben zum Arbeitsaufwand der Validierung gemacht.

\section{Ergebnisse}

Kohortenstudien mit bereits vorliegenden kardiovaskulären und metabolischen Endpunkten in Deutschland

Es konnten in Deutschland 8 populationsbasierte Kohortenstudien identifiziert werden, für die bereits kardiovaskulär-metabolische Endpunkte vorliegen (- Tab. 1; [7-14]). Drei weitere Kohortenstudien wurden aufgrund noch nicht vorliegender Endpunkte nicht aufgenommen: In der NAKO-Gesundheitsstudie, der größten derartigen Studie in Deutschland, findet zurzeit die Rekrutierung der geplanten 200.000 Teilnehmer statt [15]. In der LIFE-Studie (Leipziger Forschungszentrum für Zivilisationserkrankungen) wurde diese vor Kurzem abgeschlossen, und die Hamburger Gesundheitsstudie wurde gerade gestartet $[16,17]$.

Die Spannweite der Anzahl der Teilnehmenden in den betrachteten Studien liegt zwischen 1779 und 27.548. Die Anzahl inzidenter Fälle liegt in den Studien bei 45-1096 beim Myokardinfarkt, 60-953 beim Schlaganfall, 122-1856 beim Typ-2-Diabetes, 45-146 bei der Herzinsuffizienz und 195-667 bei der arteriellen Hypertonie (• Tab. 1).

\section{Klassifikation der kardiovaskulären und metabolischen Endpunkte}

Die Hälfte der 8 identifizierten Studien führt bei der Erfassung des Herzinfarktes keine weitere Unterteilung des Krankheitsbildes durch (• Tab. 2). In der Gutenberg-Gesundheitsstudie (GHS), in der European Prospective Investigation into Cancer and Nutrition (EPIC) in
Heidelberg und in der Heinz-NixdorfRecall-Studie (HNR) werden zusätzlich Infarktlokalisation und Unterteilung nach Vorliegen einer EKG-ST-Senkung unterschieden (STEMI/NSTEMI). In der GHS und HNR wird außerdem die Zahl der betroffenen Gefäße aufgezeichnet. Beim Schlaganfall erfasst die Hälfte der Studien, ob es sich um ischämische oder hämorrhagische Fälle handelt, und differenziert nach transienter ischämischer Attacke (TIA), prolongiertem reversiblen ischämischen neurologischen Defizit (PRIND) und Infarkt. Zwei Studien führen keine weitere Unterteilung durch. In zwei Studien (EPIC-Heidelberg, HNR) werden die sogenannten TOAST-Kriterien (Trial of Org 10172 in Acute Stroke Treatment) erfasst, dabei handelt es sich um eine ätiologische Einteilung des ischämischen Schlaganfalls [18]. Beim Typ-2-Diabetes-mellitus differenzieren die meisten Studien den Schwangerschaftsdiabetes; zwei Studien führen keine weitere Unterteilung durch. Bei der Herzinsuffizienz erfasst die Hälfte der Studien den Erhalt der Ejektionsfraktion, die „New York Heart Association“(NYHA)-Schweregradeinteilung [19] und die natriuretischen Peptide (•Tab. 2). Die Ätiologie der Herzinsuffizienz wird in zwei Studien erfasst. Bei der arteriellen Hypertonie führen die meisten Studien keine weitere Unterteilung durch, im Rahmen der Studie zur Gesundheit Erwachsener in Deutschland (DEGS) werden Hypertoniestadien unterteilt und bei der EPICPotsdam-Studie wird nach essenzieller und sekundärer Hypertonie unterschieden.

\section{Datenquellen}

Alle identifizierten Studien nutzen ein weites Spektrum an Datenquellen, um kardiovaskuläre und metabolische Krankheiten $\mathrm{zu}$ identifizieren und $\mathrm{zu}$ validieren (•Tab. 3). Dies beinhaltet die Selbstauskunft der Teilnehmenden, den Kontakt zum Hausarzt, das Anfordern von Entlassungsbriefen oder Befundkopien aus Krankenhausarchiven, den Zugriff auf Klinikinformationssysteme und den Kontakt zu Einwohnermelde- und Gesundheitsämtern. In fünf 
Bundesgesundheitsbl 2018 -61:420-431 https://doi.org/10.1007/s00103-018-2712-4

(c) Der/die Autor(en) 2018. Dieser Artikel ist eine Open-Access-Publikation.

\section{W. J. Herrmann · C. Weikert · M. Bergmann $\cdot$ H. Boeing $\cdot$ V. A. Katzke $\cdot$ R. Kaaks $\cdot$ D. Tiller $\cdot$ K. H. Greiser $\cdot$ M. Heier $\cdot$ C. Meisinger $\cdot$ C. O. Schmidt H. Neuhauser · C. Heidemann · C. Jünger · P. S. Wild · S. H. Schramm · K.-H. Jöckel · M. Dörr · T. Pischon \\ Erfassung inzidenter kardiovaskulärer und metabolischer Erkrankungen in epidemiologischen Kohortenstudien in Deutschland}

\section{Zusammenfassung}

Hintergrund. Kardiovaskuläre und metabolische Erkrankungen sind häufige Ursachen für Mortalität und Verlust von Lebensqualität in der deutschen Bevölkerung. Zur Erforschung von Risikofaktoren sind populationsbasierte Kohortenstudien notwendig. Für deren Aussagekraft ist die vollständige und valide Erfassung der Endpunkte ein zentraler Aspekt. Fragestellung. Ziel war es, derzeit in Deutschland durchgeführte populationsbasierte Kohortenstudien mit bereits erreichten inzidenten kardiovaskulären und metabolischen Erkrankungen (Herzinfarkt, Diabetes mellitus Typ 2, Schlaganfall, Herzinsuffizienz und arterielle Hypertonie) zu identifizieren und methodische Aspekte der Erfassung und Klassifikation in diesen Studien zusammenzutragen.
Methoden. Über die Arbeitsgruppe „Epidemiologie der Herz-Kreislauf- und StoffwechselErkrankungen" wurden deutsche bevölkerungsbasierte Kohortenstudien mit bereits erreichten inzidenten kardiovaskulären und metabolischen Erkrankungen identifiziert. Vertretende Personen dieser Studien wurden im Rahmen eines Workshops eingeladen, ihre Vorgehensweise vorzustellen und im Nachgang systematisch zu ergänzen und aufzubereiten.

Ergebnisse. Es wurden 8 Studien aus verschiedenen Regionen mit insgesamt 100.571 Teilnehmern und einer Altersspanne von 18-83 Jahren identifiziert. Die Selbstauskunft der Studienteilnehmenden ist die wichtigste Quelle zur Veranlassung weiterer Recherchen zur Erfassung der Endpunkte in diesen Studien. Die Selbstangaben werden in allen 8 Studien mithilfe verschiedener Informationen aus anderen Datenquellen, z. B. Unterlagen von behandelnden Ärzten oder Kliniken, ergänzt und abschließend bewertet. Diskussion. Unsere Ergebnisse zeigen die zentrale Bedeutung der Selbstauskunft und den Aufwand der erforderlichen Validierung der Endpunkte. Künftige Studien können diese Informationen in der Planung berücksichtigen und standardisieren.

Schlüsselwörter

Epidemiologie $\cdot$ Kohortenstudien .

Risikofaktoren · Herz-Kreislauf-Krankheiten ·

Metabolische Krankheiten

\section{Assessing incident cardiovascular and metabolic diseases in epidemiological cohort studies in Germany}

\section{Abstract}

Background. Cardiovascular and metabolic diseases are a major cause of mortality and loss of quality of life in Germany. Research into risk factors of these diseases requires large population-based cohort studies. Complete and accurate assessment of the incidence of cardiovascular and metabolic diseases is a key element for valid interpretation of the results from such studies.

Objective. Our aim was to identify populationbased cohort studies with incidence of cardiovascular and metabolic diseases in Germany and to summarize their methods for assessment and classification of disease endpoints, including myocardial infarction, type 2 diabetes, stroke, heart failure, and arterial hypertension.

Methods. Within the framework of a workshop, representatives of the ascertained population-based cohort studies in Germany with incidence of cardiovascular or metabolic diseases were invited to present and to systematically provide information on their methods of endpoint identification.

Results. We identified eight studies from different regions in Germany with a total of 100,571 participants, aged $18-83$ years at baseline. Self-reporting by study participants is the major source for further inquiries to assess disease endpoints in these studies. Most studies use additional data sources to verify the incidence of diseases, such as documents provided by the treating physician or hospital.

Conclusions. Our results highlight the central role of self-reporting and the efforts associated with identification and verification of disease endpoints in cohort studies. They also provide a basis for future populationbased studies that aim for standardized assessment of the incidence of cardiovascular and metabolic diseases.

Keywords Epidemiology - Cohort studies - Risk factors . Cardiovascular diseases - Metabolic diseases
Studien finden im Rahmen der Nachbeobachtungen auch Untersuchungen im Studienzentrum statt. In der Studie zur Gesundheit Erwachsener in Deutschland (DEGS) und der KooperativeGesundheitsforschung-in-der-RegionAugsburg-Studie (KORA) wird die Medikation im Rahmen der Untersuchung im Studienzentrum sehr differenziert erfasst. Abrechnungsdaten der kassenärztlichen Vereinigung werden nur in der
Study of Health in Pomerania (SHIPStudie), Krankenkassendaten werden von keiner Studie für die Ermittlung von Endpunkten verwendet.

\section{Primäre Identifizierung von inzidenten kardiovaskulären und metabolischen Krankheiten}

Alle betrachteten Kohortenstudien nutzen die Selbstauskunft als die wesentliche
Quelle, um inzidente kardiovaskuläre und metabolische Krankheiten zu identifizieren und ggf. weitere Recherchen durchzuführen. Darüber hinaus werden weitere Datenquellen genutzt, die auf ein kardiovaskuläres Ereignis hindeuten, wie beispielsweise Medikamentengebrauch und Befunde. Zur Selbstauskunft nutzen sieben Studien Fragebogen, sechs Studien persönliche Interviews und drei Studien Telefoninterviews. Dazu wer- 


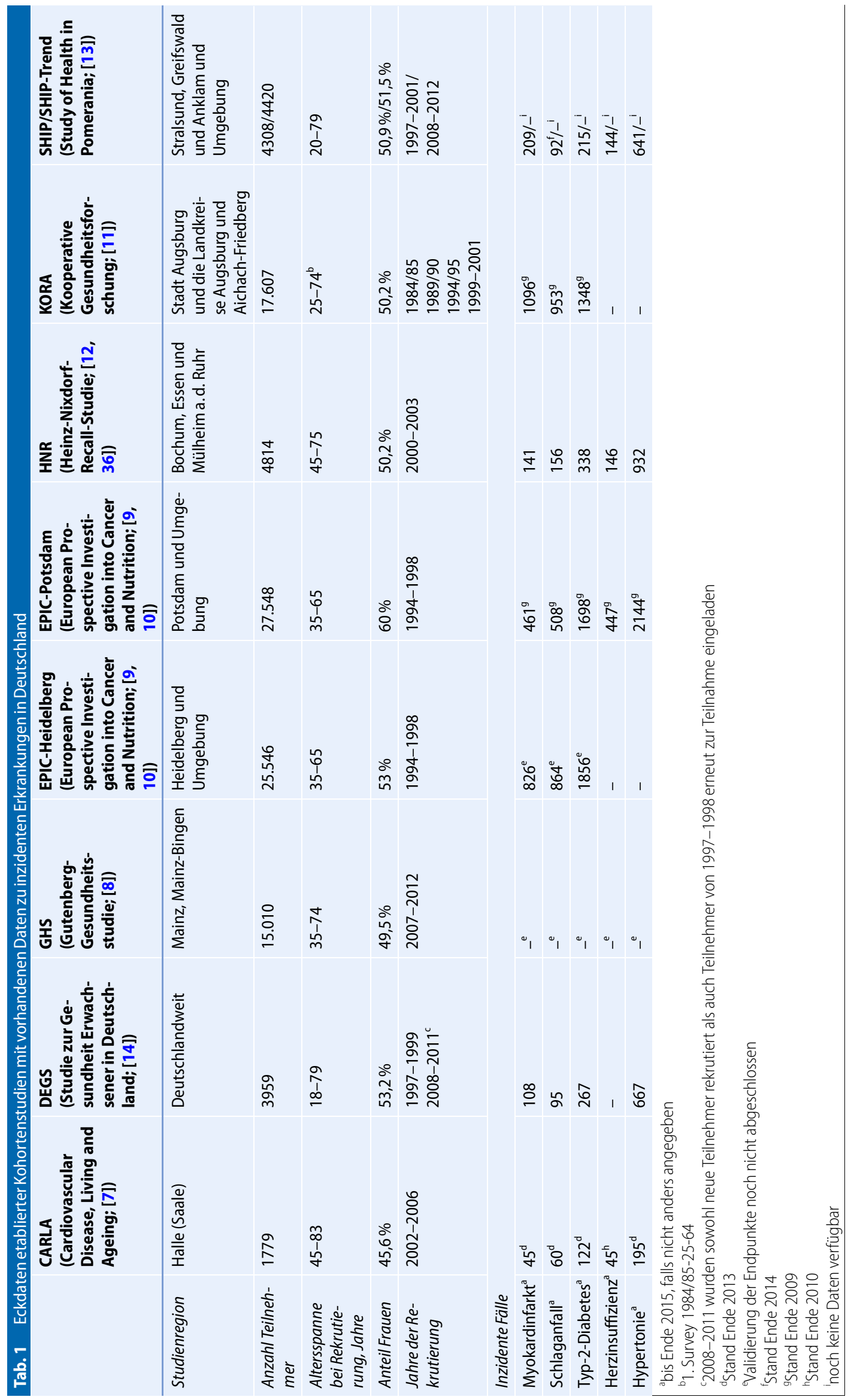




\begin{tabular}{|c|c|c|c|c|c|c|c|c|c|}
\hline Endpunkt & Unterteilung in/nach & CARLA & DEGS & GHS & EPIC-H & EPIC-P & $\begin{array}{l}\text { Heinz- } \\
\text { Nixdorf }\end{array}$ & KORA & SHIP/SHIP-T \\
\hline \multirow[t]{5}{*}{ Herzinfarkt } & Keine weitere Unterteilung & - & $x$ & - & - & $x$ & - & $x$ & $x$ \\
\hline & STEMI/NSTEMI & - & - & $x$ & $x$ & - & $x$ & - & - \\
\hline & $\begin{array}{l}\text { Lokalisation (z. B. Vorderwand- } \\
\text { infarkt) }\end{array}$ & - & - & $x$ & $x$ & - & $x$ & - & - \\
\hline & $\begin{array}{l}\text { Anzahl der Gefäße (z. B. Drei- } \\
\text { gefäßerkrankung) }\end{array}$ & - & - & $x$ & - & - & $x$ & - & - \\
\hline & Andere Unterteilung & $X^{a}$ & - & $X^{a}$ & - & $X^{a, b}$ & $X^{b}$ & - & $X^{a}$ \\
\hline \multirow[t]{5}{*}{ Schlaganfall } & Keine weitere Unterteilung & - & $x$ & - & - & - & - & - & $x$ \\
\hline & Ischämisch/hämorrhagisch & - & - & $x$ & $x$ & $x$ & $x$ & $x$ & - \\
\hline & TIA, PRIND, Hirninfarkt & $x$ & - & $x$ & $x$ & $x$ & $x$ & $x$ & - \\
\hline & TOAST-Kriterien & - & - & - & $x$ & - & $X-$ & - & - \\
\hline & Andere Unterteilung & - & - & - & - & - & - & - & - \\
\hline \multirow{3}{*}{$\begin{array}{l}\text { Diabetes } \\
\text { mellitus Typ } 2\end{array}$} & Keine weitere Unterteilung & $x$ & - & - & - & - & - & $x$ & - \\
\hline & Schwangerschaftsdiabetes & - & $x$ & $x$ & $x$ & $x$ & $x$ & - & $x$ \\
\hline & Andere Unterteilung & - & $x^{c}$ & $X^{d}$ & $x^{e}$ & $x^{f}$ & $x^{e}$ & - & $x^{g}$ \\
\hline \multirow{6}{*}{$\begin{array}{l}\text { Herzinsuffi- } \\
\text { zienz }\end{array}$} & Keine weitere Unterteilung & - & $x$ & - & - & $x$ & - & - & - \\
\hline & $\begin{array}{l}\text { Reduzierte/erhaltene Ejektions- } \\
\text { fraktion }\end{array}$ & $x$ & - & $x$ & - & $x$ & $x$ & - & $x$ \\
\hline & $\begin{array}{l}\text { Ätiologie (ischämisch, nicht- } \\
\text { ischämisch, Kardiomyopathie, } \\
\text { valvuläre, sonstige) }\end{array}$ & - & - & $(\mathrm{X})$ & - & - & $x$ & - & $(X)$ \\
\hline & Schweregradeinteilung nach NYHA & $\mathrm{x}$ & - & $\mathrm{x}$ & - & $x$ & $x$ & - & $x$ \\
\hline & $\begin{array}{l}\text { Natriuretische Peptide (BNP, NT- } \\
\text { proBNP) }\end{array}$ & $x$ & - & $x$ & - & $--^{h}$ & $x$ & - & $x$ \\
\hline & Andere Unterteilung & - & - & $x^{i}$ & - & - & - & - & - \\
\hline \multirow{2}{*}{$\begin{array}{l}\text { Arterielle } \\
\text { Hypertonie }\end{array}$} & Keine weitere Unterteilung & $x$ & - & - & - & - & - & - & $x$ \\
\hline & Andere Unterteilung & $x^{j}$ & $x^{j}$ & $x^{j}$ & - & $x^{k}$ & $x^{\prime}$ & - & - \\
\hline \multicolumn{10}{|c|}{ 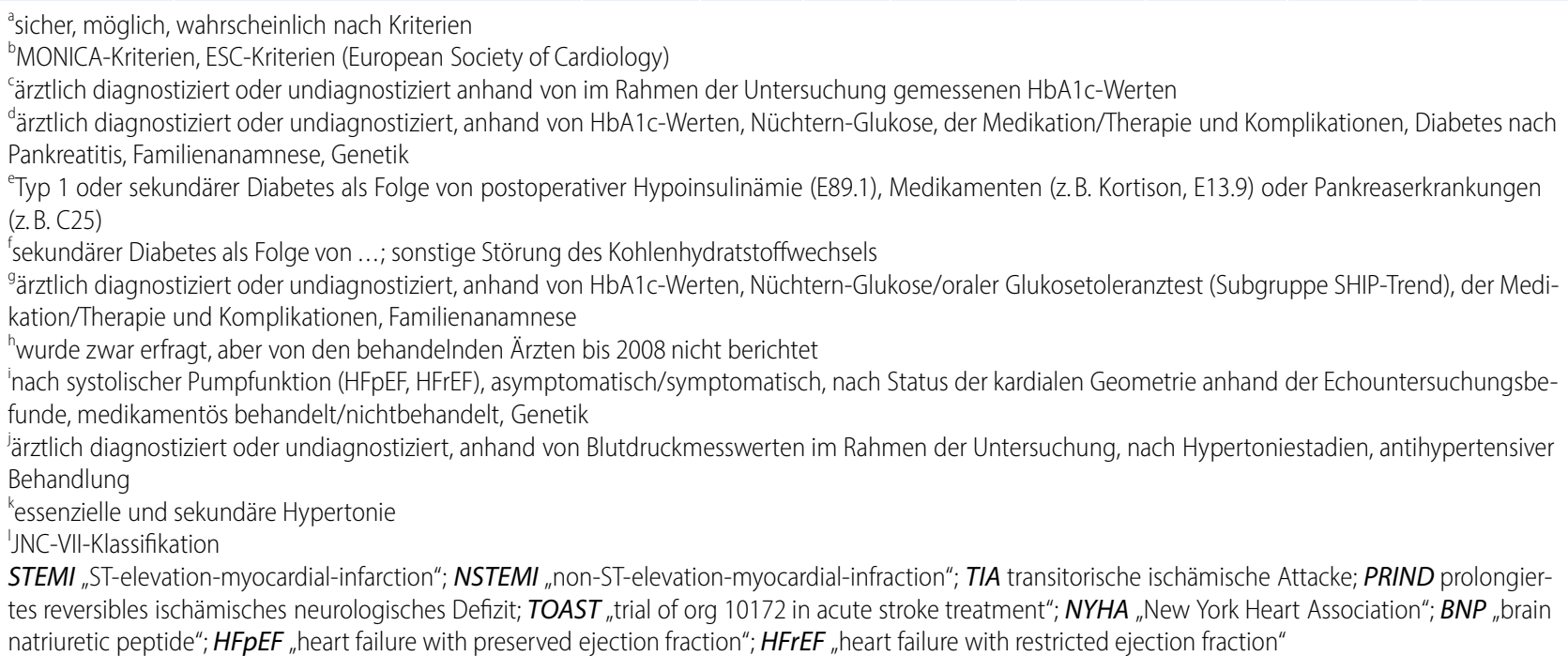 } \\
\hline
\end{tabular}

den die Teilnehmenden meist gezielt nach dem Eintreten der betreffenden Erkrankung, des Ereignisses oder der Diagnose befragt. Andererseits wird bei einigen Erkrankungen zur Steigerung der Sensitivität auch nach Symptomen,
Medikamenten oder Therapieverfahren gefragt.

Wenn Teilnehmende über den Postweg nicht erreichbar sind, wird in allen Studien das jeweilige Einwohnermeldeamt kontaktiert, um zu erfahren, ob sie verzogen oder verstorben sind. In den meisten Studien (mit Ausnahme von HNR und DEGS) erfolgt eine Abfrage beim Einwohnermeldeamt darüber hinaus regelmäßig für alle Teilnehmenden. Wenn Teilnehmende verstorben sind, so 


\begin{tabular}{|c|c|c|c|c|c|c|c|c|}
\hline & CARLA & DEGS & GHS & EPIC-H & EPIC-P & $\begin{array}{l}\text { Heinz- } \\
\text { Nixdorf }\end{array}$ & KORA & $\begin{array}{l}\text { SHIP/ } \\
\text { SHIP-TREND }\end{array}$ \\
\hline \multicolumn{9}{|l|}{ Selbstauskunft } \\
\hline Erkrankung/Diagnose & $x$ & $x$ & $x$ & $x$ & $x$ & $x$ & $x$ & $x$ \\
\hline Medikamente & $x$ & $x$ & $x$ & $x$ & $x$ & $x$ & $x$ & $x$ \\
\hline Symptome & $x$ & - & $x$ & - & $x$ & $x$ & $x$ & $x$ \\
\hline Hausärzte & $x$ & - & $x$ & $x$ & $x$ & $x$ & $x$ & $x$ \\
\hline Entlassungsbriefe anfordern & $x$ & - & $x$ & $x$ & $x$ & $x$ & $x$ & $x$ \\
\hline Zugriff auf Klinikinformationssystem & - & - & $x$ & $x$ & - & $x$ & $x$ & $x$ \\
\hline $\begin{array}{l}\text { Anfordern von Befundkopien aus Krankenhaus- } \\
\text { archiven o. } \ddot{\text { A. }}\end{array}$ & - & - & $x$ & $x$ & $x$ & $x$ & $x$ & - \\
\hline Meldeamt & $x$ & $x$ & $x$ & $x$ & $x$ & $x$ & $x$ & $x$ \\
\hline Gesundheitsamt & $x$ & $x$ & $x$ & $x$ & - & $x$ & $x$ & $x$ \\
\hline \multicolumn{9}{|l|}{ Abrechnungsdaten } \\
\hline Kassenärztliche Vereinigungen & - & - & - & - & - & - & - & $x$ \\
\hline Krankenkassen & - & - & - & - & - & - & - & - \\
\hline $\begin{array}{l}\text { Untersuchung im Untersuchungszentrum (einschl. } \\
\text { Laborwertbestimmung) }\end{array}$ & $x$ & $x$ & $x$ & - & - & $x$ & $x$ & $x$ \\
\hline Andere & $x^{a}$ & - & $x^{b}$ & - & $X^{c}$ & $x^{c}$ & $x^{a}$ & - \\
\hline
\end{tabular}

wird in allen Studien beim Gesundheitsamt angefragt, um Informationen zu Todesursachen von den Totenscheinen nutzen zu können.

\section{Validierung der Daten}

Nach der primären Identifikation inzidenter Fälle werden verschiedene Datenquellen hinzugezogen, um die Diagnose zu prüfen oder ggf. zusätzliche inzidente Fälle zu finden. Eine - mit Ausnahme der DEGS-Studie - in allen Kohortenstudien genutzte Datenquelle sind die behandelnden Ärzte der Studienteilnehmenden. Eine typische Vorgehensweise ist die der EPIC-Potsdam- und der Cardiovascular-Disease-Living-andAgeing-Studie (CARLA; [7, 10]): Zur Validierung wird den behandelnden Ärzten ein Kurzfragebogen zugesandt mit der Bitte, die Diagnose zu bestätigen und Fragen zu den zugrunde liegenden Diagnosekriterien zu beantworten. Eine Herausforderung ist auch hier das fragmentierte Gesundheitswesen in Deutschland, da nicht alle Befunde bei einem Arzt zusammenlaufen. Deshalb werden die Teilnehmenden in fast allen Kohortenstudien (bis auf DEGS) auch gebeten, Namen und Adresse des Hausarztes oder der diagnosestellenden Ärztin anzugeben. Neben Informationen aus dem ambulanten Sektor werden auch Entlassungsbriefe aus Krankenhäusern und in der HNRStudie auch Originalbefunde aus Krankenhausarchiven angefordert.

Darüber hinausgehend besteht bei EPIC-Heidelberg, der HNR-Studie und der GHS die Möglichkeit, auf das Klinikinformationssystem der Universitätsklinik zuzugreifen, um ausgehend von der Selbstangabe einer Erkrankung gezielt Befunde und Briefe zur Validierung einzusehen. In SHIP sind kardiovaskuläre Entlassungsdiagnosen von an der Universitätsmedizin behandelten teilnehmenden Personen über das Krankenhausinformationssystem einsehbar. In allen Studien werden bei verstorbenen Teilnehmenden darüber hinaus die Angaben aus dem Totenschein zur Validierung mithinzugezogen.

Die abschließende Beurteilung und Klassifikation der Studienteilnehmenden hinsichtlich der Endpunkte erfolgt in den meisten Studien durch einen Studienarzt oder durch festgelegte Algorithmen. Dieser Ablauf sieht beispielsweise in der CARLA-Studie wie folgt aus: Als inziden- ter Typ-2-Diabetiker wird klassifiziert, wer entweder aktuell antidiabetische Medikamente einnimmt, eine über den Hausarzt verifizierte Selbstangabe im Interview getätigt hat oder erhöhte Laborparameter aufweist, welche anschließend vom Hausarzt als vorliegender Diabetes bestätigt werden. In einigen wenigen Studien werden auch Endpunktkomitees eingesetzt; beispielsweise in der HNR-Studie: Zur Validierung kardiologischer Endpunkte und unklarer Todesfälle treffen sich jährlich Fachleute aus den Bereichen Kardiologie, Epidemiologie und Statistik und sichten die zusammengetragenen Befunde. Zur Validierung der Schlaganfälle werden Arztbriefe und relevante Befunde in Kopie an einen Neurologen des Universitätsklinikums Essen und an einen externen Neurologen gesendet.

Dabei ist der gesamte Prozess zeit- und ressourcenaufwendig. So summiert sich beispielhaft pro Jahr in der HNR-Studie der Aufwand des Einholens und Beurteilens der Originalbefunde auf knapp 1000 Arbeitsstunden und in EPIC-Heidelberg auf 300-500 zu beurteilende Befunde. 


\begin{tabular}{|c|c|c|c|c|c|c|c|c|c|}
\hline & & CARLA & DEGS & GHS & EPIC-H & EPIC-P & $\begin{array}{l}\text { Heinz- } \\
\text { Nixdorf }\end{array}$ & KORA & $\begin{array}{l}\text { SHIP/ } \\
\text { SHIP-TREND }\end{array}$ \\
\hline \multirow[t]{15}{*}{ Herzinfarkt } & \multicolumn{9}{|l|}{ Selbstauskunft } \\
\hline & Erkrankung/Diagnose & $x$ & $x$ & $x$ & $x$ & $(X)$ & $x$ & $x$ & $x$ \\
\hline & Medikamente & - & - & - & - & - & $x$ & - & $x$ \\
\hline & Symptome & - & - & $x$ & - & - & $x$ & - & $x$ \\
\hline & \multicolumn{9}{|l|}{ Berücksichtigte Diagnostik zur Validierung } \\
\hline & Diagnose durch behandelnden Arzt & $x$ & - & $x$ & $x$ & $x$ & $x$ & $x$ & $x$ \\
\hline & Echokardiographie & - & - & $x$ & $x$ & $x^{a}$ & $x$ & - & $x$ \\
\hline & EKG-Veränderung & $x$ & - & $x$ & $x$ & $X^{a}$ & $x$ & $(X)$ & $x$ \\
\hline & Herzenzyme & - & - & $x$ & $x$ & $X^{a}$ & $x$ & $(X)$ & $x$ \\
\hline & Koronarangiographie & - & - & $x$ & $x$ & - & $x$ & $(X)$ & - \\
\hline & MRT & - & - & $x$ & - & - & $(X)$ & - & $x$ \\
\hline & \multicolumn{9}{|l|}{ Beurteilung der Befunde } \\
\hline & Algorithmus & $x$ & - & - & $x$ & - & - & $x$ & $x$ \\
\hline & Studienarzt & - & - & - & $x$ & $x$ & - & $x$ & $x$ \\
\hline & Endpunktkomitee & - & - & $x$ & - & - & $x$ & - & - \\
\hline \multirow[t]{12}{*}{ Schlaganfall } & \multicolumn{9}{|l|}{ Selbstauskunft } \\
\hline & Erkrankung/Diagnose & $x$ & $x$ & $x$ & $x$ & $(X)$ & $x$ & $x$ & $x$ \\
\hline & Medikamente & - & - & - & - & - & - & - & - \\
\hline & Symptome & - & - & - & - & - & $(\mathrm{X})$ & - & - \\
\hline & \multicolumn{9}{|l|}{ Berücksichtigte Diagnostik zur Validierung } \\
\hline & Diagnose durch behandelnden Arzt & $x$ & - & $x$ & $x$ & $x$ & $x$ & $x$ & $x$ \\
\hline & $\mathrm{MRT} / \mathrm{CT}$ & - & - & $x$ & $(X)$ & $x^{a}$ & $x$ & $(X)$ & $x$ \\
\hline & Zusatzdiagnostik & - & - & $x$ & - & $x^{a}$ & - & - & - \\
\hline & \multicolumn{9}{|l|}{ Beurteilung der Befunde } \\
\hline & Algorithmus & - & - & - & $x$ & - & - & - & $x$ \\
\hline & Studienarzt & - & - & - & $x$ & $x$ & - & $x$ & $x$ \\
\hline & Endpunktkomitee & - & - & $x$ & - & - & $x$ & - & - \\
\hline
\end{tabular}

\section{Herzinfarkt}

Potenzielle Herzinfarkte werden in allen Kohortenstudien primär über die Selbstauskunft der Teilnehmenden identifiziert (•Tab. 4). Angaben auf den Totenscheinen sind eine weitere primäre Informationsquelle. Zur Validierung wird in allen Studien (bis auf DEGS) die Diagnose der behandelnden Ärzte herangezogen, wobei rund die Hälfte auch diagnostische Maßnahmen heranziehen, wie bspw. Veränderungen im EKG. Vier Studien nutzen einen Algorithmus zur Beurteilung der Befunde, drei einen Studienarzt und eine ein Endpunktkomitee. Beispielhaft verwenden die KORAStudie und die HNR-Studie den MONICA-Algorithmus [5] und die HNR-
Studie die ESC-Kriterien (European Society of Cardiology). In der CARLAStudie wird der Minnesota-Code aus Basis- und Follow-up-EKG verwendet, um per Computeralgorithmus inzidente Infarkte festzustellen. Daten aus EPICHeidelberg bzw. EPIC-Potsdam zeigen, dass die Selbstauskunft der Diagnose Herzinfarkt in $67 \%$ bzw. $64 \%$ bestätigt und in $29 \%$ bzw. $35 \%$ falsifiziert werden konnte und in $4 \%$ bzw. $1 \%$ nicht aufklärbar war.

\section{Diabetes mellitus Typ 2}

Potenzieller Typ-2-Diabetes wird in allen Kohortenstudien primär über die Selbstauskunft über den Eintritt der Krankheit, dessen Diagnose oder durch die Einnah- me spezifischer Medikamente identifiziert (•Tab. 5). In sieben der Studien erfolgt die Validierung der Diagnose über den behandelnden Arzt. Diagnostische Kriterien werden in rund der Hälfte der Studien berücksichtigt. In vier Studien wird ein Algorithmus angewendet, um die Befunde zu beurteilen, und in dreien wird ein Studienarzt zurate gezogen. Beispielhaft ist der in der KORA-Studie verwendete Algorithmus: Diabetes mellitus Typ 2 wurde durch den Hausarzt bestätigt ODER Einnahme von Antidiabetika und/oder Insulin (ausgenommen Metformin) ODER Einnahme von Metformin und Selbstangabe Diabetes.

Daten aus EPIC-Heidelberg bzw. EPIC-Potsdam zeigen, dass die Selbstauskunft Diabetes mellitus Typ 2 in $85 \%$ 


\begin{tabular}{|c|c|c|c|c|c|c|c|c|}
\hline & CARLA & DEGS & GHS & EPIC-H & EPIC-P & $\begin{array}{l}\text { Heinz- } \\
\text { Nixdorf }\end{array}$ & KORA & $\begin{array}{l}\text { SHIP/ } \\
\text { SHIP-TREND }\end{array}$ \\
\hline \multicolumn{9}{|l|}{ Selbstauskunft } \\
\hline Erkrankung/Diagnose & $x$ & $x$ & $x$ & $x$ & $(X)$ & $x$ & $x$ & $x$ \\
\hline Medikamente & $x$ & $x$ & $x$ & $x$ & - & $x$ & $x$ & $x$ \\
\hline Symptome & - & - & - & - & - & $x$ & - & - \\
\hline \multicolumn{9}{|c|}{ Berücksichtigte Diagnostik zur Validierung } \\
\hline Diagnose durch behandelnden Arzt & $x$ & - & $x$ & $x$ & $x$ & $x$ & $x$ & $x$ \\
\hline Nüchtern-Glukose & - & - & $x$ & - & $X^{a}$ & $x$ & $(X)^{b}$ & $x$ \\
\hline Glukosetoleranztest & - & - & - & - & $x^{a}$ & $(X)^{b}$ & $(X)^{\mathrm{b}}$ & $x$ \\
\hline $\mathrm{HbA1c}$ & $(X)$ & $X^{c}$ & $x$ & - & $x^{a}$ & $x$ & - & $x$ \\
\hline Urin-Glukose & - & - & - & - & $X^{a}$ & $x$ & - & - \\
\hline Nichtnüchtern-Glukose & $(X)$ & - & - & - & - & $x$ & - & $x$ \\
\hline \multicolumn{9}{|l|}{ Beurteilung der Befunde } \\
\hline Algorithmus & $x$ & - & $x$ & - & - & - & $x$ & $x$ \\
\hline Studienarzt & - & - & - & $x$ & $x$ & - & $x$ & $x$ \\
\hline Endpunktkomitee & - & - & - & - & - & - & - & - \\
\hline Andere & - & - & - & - & $X^{d}$ & $X^{d}$ & - & - \\
\hline \multicolumn{9}{|c|}{$\begin{array}{l}\text { Kriterien: } X \text { notwendiges Kriterium, }(X) \text { mitberücksichtigtes Kriterium } \\
\text { awird auf dem Arztbogen mit abgefragt } \\
\text { btrifft nur für einen Teil der Kohorte zu } \\
\text { cnicht zur Validierung, sondern zur Identifizierung unerkannter Fälle } \\
{ }^{d} \text { Wissenschaftler mit Fragestellung }\end{array}$} \\
\hline
\end{tabular}

bzw. $94 \%$ bestätigt, in $8 \%$ bzw. $5 \%$ falsifiziert wurde und in $7 \%$ bzw. $1 \%$ nicht aufklärbar war.

\section{Schlaganfall}

Potenzieller Schlaganfall wird in allen Kohortenstudien im Wesentlichen über die Selbstauskunft der Teilnehmenden identifiziert; Angaben zu Medikamenten oder Symptomen spielen hingegen keine Rolle (-Tab.4). Angaben auf den Totenscheinen sind eine weitere Informationsquelle. Zur Validierung nutzen sechs Studien die Diagnose der behandelnden Ärzte. MRT- oder CT-Aufnahmen werden in sechs Studien zur Validierung angefordert. Drei Studien wenden einen Algorithmus an, um die Befunde zu beurteilen, drei ziehen einen Studienarzt zurate und zwei haben ein Endpunktkomitee.

Daten aus EPIC-Heidelberg zeigen, dass die Selbstauskunft Schlaganfall in $64 \%$ validiert, in $31 \%$ falsifiziert und in $5 \%$ nicht geklärt werden konnte. In EPIC-Potsdam werden auch Selbstangaben zu transitorischen ischämischen Attacken (TIA) verifiziert. Daraus ergaben sich folgende Anteile: Schlaganfall: $70 \%$ als Schlaganfall bestätigt, $18 \%$ als TIA, $11 \%$ falsifiziert und $1 \%$ unklar. TIA: $4 \%$ bestätigt als Schlaganfall, $31 \%$ als TIA, $46 \%$ falsifiziert und $19 \%$ unklar.

\section{Herzinsuffizienz}

Potenzielle Herzinsuffizienz wird in sieben der acht Studien über die Selbstauskunft der Teilnehmenden identifiziert (• Tab. 6). Drei Studien stützen sich zusätzlich auf Medikamentenangaben und vier auf die Angabe von Symptomen. In fünf Studien wird die Diagnose der behandelnden Ärzte zur Validierung berücksichtigt. Ergebnisse aus apparativen Untersuchungen werden in rund der Hälfte der Studien berücksichtigt. In drei Studien erfolgt die Beurteilung der Befunde über einen Algorithmus, eine Studie zieht einen Studienarzt, eine ein Endpunktkomitee zurate.

Ein beispielhafter Algorithmus ist jener der CARLA-Studie, der auf Empfehlungen der Europäischen Kardiologischen Gesellschaft (ESC) beruht und Symptome und echokardiographische Zeichen heranzieht [20]. Als weiteres
Kriterium zur Bestimmung der Herzinsuffizienz mit erhaltener Ejektionsfraktion (HFNEF) wurde das NT-proBNP als diagnostischer Marker einbezogen [21].

Daten aus EPIC-Potsdam zeigen, dass die Selbstauskunft Herzinsuffizienz einen niedrigen positiven prädiktiven Wert hat: Nur 23,5\% wurden validiert, 76,1 \% wurden falsifiziert und $0,4 \%$ blieben unklar.

\section{Arterielle Hypertonie}

Die arterielle Hypertonie wird in sieben von 8 Studien über Selbstangaben des Teilnehmers erfasst (• Tab. 6). In 5 Studien werden auch Medikamente und in einer Studie Symptome herangezogen. In 4 Studien wird die Diagnose durch den behandelnden Arzt validiert. In 6 Studien werden zudem die Blutdruckwerte aus der Nachuntersuchung im Untersuchungszentrum berücksichtigt. In 5 Studien wird ein Algorithmus zur Beurteilung der Befunde angewendet. Eine Studie zieht einen Studienarzt zurate. Ein Endpunktkomitee wird in keiner Studie eingesetzt.

Beispielhaft ist der Algorithmus aus der DEGS-Studie, bei dem eine Hyperto- 


\begin{tabular}{|c|c|c|c|c|c|c|c|c|c|}
\hline & & CARLA & DEGS & GHS & EPIC-H & EPIC-P & $\begin{array}{l}\text { Heinz- } \\
\text { Nixdorf }\end{array}$ & KORA & $\begin{array}{l}\text { SHIPI } \\
\text { SHIP-TREND }\end{array}$ \\
\hline \multirow[t]{17}{*}{ Herzinsuffizienz } & \multicolumn{9}{|l|}{ Selbstauskunft } \\
\hline & Erkrankung/Diagnose & $x$ & $x$ & $x$ & $\mathrm{X}$ & $(X)$ & $x$ & - & $x$ \\
\hline & Medikamente & - & - & $x$ & - & - & $x$ & - & $x$ \\
\hline & Symptome & $x$ & - & $x$ & - & - & $x$ & - & $x$ \\
\hline & \multicolumn{9}{|c|}{ Berücksichtigte Diagnostik zur Validierung } \\
\hline & Diagnose durch behandelnden Arzt & $x$ & - & $x$ & - & $x$ & $x$ & - & $X^{c}$ \\
\hline & Echokardiographie & $x$ & - & $x$ & - & $X^{a}$ & $x$ & - & $x$ \\
\hline & Klinische Untersuchung & - & - & $x$ & - & $X^{a}$ & $x$ & - & $x$ \\
\hline & MRT & - & - & $x$ & - & - & $x$ & - & $x$ \\
\hline & NT-proBNP/BNP & $x$ & - & $x$ & - & $X^{a}$ & $x$ & - & $x$ \\
\hline & EKG & $x$ & - & - & - & $x^{a}$ & $x$ & - & - \\
\hline & Röntgen-Thorax & - & - & - & - & $X^{a}$ & - & - & - \\
\hline & \multicolumn{9}{|l|}{ Beurteilung der Befunde } \\
\hline & Algorithmus & $x$ & - & $x$ & - & - & - & - & $x$ \\
\hline & Studienarzt & - & - & - & - & - & - & - & $x$ \\
\hline & Endpunktkomitee & - & - & $x$ & - & - & - & - & - \\
\hline & Andere & - & - & - & - & - & $x^{b}$ & - & - \\
\hline \multirow{11}{*}{$\begin{array}{l}\text { Arterielle } \\
\text { Hypertonie }\end{array}$} & \multicolumn{9}{|l|}{ Selbstauskunft } \\
\hline & Erkrankung/Diagnose & $x$ & $(X)$ & $x$ & $x$ & $(X)$ & $x$ & - & $x$ \\
\hline & Medikamente & $x$ & $x$ & $x$ & - & - & $x$ & - & $x$ \\
\hline & Symptome & - & - & - & - & - & $x$ & - & - \\
\hline & \multicolumn{9}{|c|}{ Berücksichtigte Diagnostik zur Validierung } \\
\hline & Diagnose durch behandelnden Arzt & - & - & $x$ & - & $x$ & $x$ & - & $x$ \\
\hline & $\begin{array}{l}\text { Blutdruckwerte bei Nachuntersu- } \\
\text { chung }\end{array}$ & $x$ & $x^{d}$ & $x$ & - & - & $x$ & - & $x$ \\
\hline & \multicolumn{9}{|l|}{ Beurteilung der Befunde } \\
\hline & Algorithmus & $x$ & $x$ & $x$ & - & - & $x$ & - & $x$ \\
\hline & Studienarzt & - & - & - & - & $x$ & - & - & $x$ \\
\hline & Endpunktkomitee & - & - & - & - & - & - & - & - \\
\hline \multicolumn{10}{|c|}{$\begin{array}{l}\text { Kriterien: } X \text { notwendiges Kriterium, }(X) \text { mitberücksichtigtes Kriterium } \\
\text { awird auf dem Arztbogen mit abgefragt } \\
\text { bWissenschaftler mit Fragestellung } \\
\text { 'ab SHIP-2/SHIP-TREND-0 } \\
\text { 'nicht zur Validierung, sondern zur Identifizierung von Fällen } \\
\text { MRT Magnetresonanztomographie; } B N P_{\text {"brain natriuretic peptide"; EK }}\end{array}$} \\
\hline
\end{tabular}

nie basierend auf Messwerten, Medikation und Selbstauskunft zu Arztdiagnosen bei der Untersuchung definiert wird: (Blutdruckmesswert $\quad \geq 140 / 90 \mathrm{mmHg}$ ) ODER (Einnahme Medikamente der ATC-Gruppen C02, C03, C07, C08; C09 UND selbstberichtete Arztdiagnose Hypertonie jemals).

Daten aus EPIC-Potsdam zeigen, dass die Selbstauskunft Bluthochdruck in $88,9 \%$ validiert, in $10,2 \%$ falsifiziert und in $0,9 \%$ weder validiert noch falsifiziert werden konnte.

\section{Diskussion}

Es wurden 8 populationsbasierte Kohortenstudien identifiziert, in denen nach der Basisuntersuchung im Verlauf der Nachbeobachtung bereits eine große Zahl an inzidenten kardiovaskulären und metabolischen Erkrankungen vorliegt. Wichtigste Quelle für die primäre Erfassung dieser Endpunkte ist die Selbstauskunft der Studienteilnehmenden. In fast allen Studien werden diese Angaben durch Informationen aus anderen Datenquellen ergänzt bzw. be- stätigt, wobei die behandelnden Ärzte die wichtigste bzw. am häufigsten genutzte Quelle sind. Diese Ergebnisse zeigen die große Bedeutung der Selbstauskunft, aber auch den Aufwand, der mit der Validierung inzidenter Erkrankungen in Kohortenstudien einhergeht.

\section{Erhebung und Klassifikation von kardiovaskulären und metabolischen Endpunkten}

Entscheidend für die Erhebung und Klassifikation von Endpunkten in epi- 
demiologischen Studien ist einerseits möglichst alle neu aufgetretenen Fälle zu erfassen (hohe Sensitivität) und andererseits zusätzlich über eine hohe Spezifität einen hohen positiven prädiktiven Wert sicherzustellen. Eine Fehlklassifikation von Studienteilnehmern sollte weitestgehend vermieden werden, da hierdurch Risikozusammenhänge verzerrt werden könnten [22]. Da in populationsbasierten Kohortenstudien der Anteil von Personen mit neu aufgetretenen kardiovaskulären und metabolischen Endpunkten im Vergleich zur Ausgangspopulation in der Regel gering ist, treten mögliche Verzerrungen vor allem durch fälschlich als krank und weniger durch fälschlich als nichterkrankt klassifizierte Personen auf [23]. Dies spricht dafür, dass die Selbstauskunft zur Identifizierung potenzieller Fälle möglichst sensitiv sein sollte, während die Validierung dieser potenziellen Fälle möglichst spezifisch erfolgen sollte.

\section{Primäre Identifizierung von inzidenten kardiovaskulären und metabolischen Krankheiten}

Alle betrachteten Kohortenstudien nutzen die Selbstauskunft der Teilnehmenden, um inzidente kardiovaskuläre und metabolische Erkrankungen zu identifizieren. Der Vorteil besteht darin, dass aufgrund der in Deutschland fragmentierten medizinischen Versorgung die Studienteilnehmenden oftmals als Einzige einen guten Überblick über ihre diagnostizierten Erkrankungen haben und die Zeit seit der Basisuntersuchung bzw. letzten Nachbeobachtung überblicken können. Bei großen Studien bieten Fragebögen die Möglichkeit, solche Informationen schnell und effizient zu sammeln. Nachteile der Selbstauskunft sind, dass sie vom Erinnerungs-, Verständnis- und Wiedergabevermögen der Studienteilnehmenden sowie von ihrem Inanspruchnahmeverhalten abhängen. Daher wird die Selbstauskunft in der Regel nachfolgend mit weiteren Informationsquellen abgeglichen, um die Validität der Diagnose zu erhöhen. Dies steht im Gegensatz zur Erfassung kognitiver Störungen und demenzieller Erkrankungen [24], bei denen Untersuchungen und Testung der
Studienteilnehmenden statt der Selbstauskunft eine zentrale Rolle spielen.

Bei verstorbenen Studienteilnehmenden spielen die Gesundheitsämter für die primäre Erfassung von inzidenten (letalen) kardiovaskulären und metabolischen Krankheiten eine wichtige Rolle. Die Validität der dokumentierten Todesursachen auf Totenscheinen ist jedoch häufig eingeschränkt $[25,26]$. Die Verbesserung der Todesursachencodierung durch das elektronische Codiersystem „Iris“ wird in DEGS genutzt [27]; mithilfe von Iris wird dabei elektronisch das dem Tod zugrunde liegende Grundleiden bestimmt. In der CARLA- und der HNRStudie werden alle Totenscheine noch einmal vom Statistischen Landesamt nachträglich codiert.

Abrechnungsdaten der kassenärztlichen Vereinigungen und gesetzlichen Krankenversicherungen spielen bei der Identifikation inzidenter kardiovaskulärer und metabolischer Erkrankungen bisher nur eine untergeordnete Rolle und werden nur in der SHIP-Studie verwendet [28]. Ihre Nutzung beinhaltet eigene methodische Schwierigkeiten und sollte Validierungsmechanismen beinhalten [28, 29].

In einigen Kohortenstudien finden auch während der Nachbeobachtung erneute Untersuchungen im Studienzentrum statt. In der Regel reichen diese jedoch nicht für eine Klassifikation von Teilnehmenden hinsichtlich der interessierenden Endpunkte aus. Zum einen sind sie meist für eine Diagnosestellung nicht umfangreich genug, zum anderen liegen zwischen den Untersuchungen meist lange Zeiträume, sodass eine Bestimmung des Eintrittsdatums der Diagnose nicht genau möglich ist. Darüber hinaus folgen nicht alle Teilnehmenden einer Einladung ins Untersuchungszentrum und dies kann mit den Endpunkten zusammenhängen, die von Interesse sind. Ein weiteres Problem ist die zu geringe Sensitivität, da sich pathologische Veränderungen in technischen Untersuchungen und Bildgebung, z.B. nach Schlaganfall, wieder zurückbilden können. Jedoch können die Untersuchungsergebnisse in $\mathrm{Zu}$ sammenschau mit anderen Daten zur Validierung von Diagnosen genutzt wer- den, beispielsweise EKG-Veränderungen nach Herzinfarkt. Sie können aber auch zur primären Identifizierung von bisher unbekannten Erkrankungen wie Typ-2Diabetes oder Hypertonie führen.

Register sind für kardiovaskuläre Erkrankungen nicht deutschlandweit implementiert; regionale Ausnahmen sind beispielsweise die epidemiologisch geprägten Herzinfarktregister Sachsen-Anhalt und KORA $[4,5,30]$. Register werden in den beschriebenen Studien zur primären Identifizierung von inzidenten kardiovaskulären und metabolischen Krankheiten nicht verwendet, allerdings in KORA zur Validierung genutzt.

\section{Validierung der Daten}

Hinsichtlich der Validierung lassen sich verschiedene Vorgehensweisen unterscheiden: zum einen die direkte Validierung der Diagnose und strukturierte Abfrage von Informationen über behandelnde Ärzte. Diese Vorgehensweise wird von fast allen identifizierten Studien (außer DEGS, HNR und GHS) angewendet. Dafür muss der behandelnde Arzt bekannt sein. Allerdings ist nicht immer klar, ob dieser die Diagnose selbst gestellt oder von anderen übernommen hat. Die zugrunde liegende Diagnostik kann bei diesem Vorgehen oftmals nicht geklärt werden. Auch kann unklar sein, ob die Antwort zur Validierung von dem Arzt selbst oder vom angestellten Fachpersonal kommt. Die Studien sind auf die Unterstützung der behandelnden Ärzte angewiesen.

Der Vorteil des Abfragens konkreter Diagnosekriterien liegt darin, dass besser eingeschätzt werden kann, auf welchen Kriterien eine Diagnose beruht. Sollten sich diese im Laufe der Studie ändern, wäre gegebenenfalls eine Anpassung möglich. In epidemiologischen Analysen könnte der „Wahrscheinlichkeitsgrad" für das Bestehen einer Diagnose berücksichtigt werden.

Eine andere weitaus aufwendigere Vorgehensweise ist das Einholen von Originalbefunden von behandelnden Ärzten oder dem Krankenhaus, die dann von der Studienleitung oder einem Komitee zur Validierung der Diagnose verwendet werden. Diese Methode 
wird beispielsweise in der HNR-Studie angewendet. In EPIC-Heidelberg und GHS werden Unterlagen aus Kliniken angefordert.

In der Zusammenschau aller Informationen erfolgt dann durch Algorithmen, Studienärzte oder ein Endpunktkomitee eine abschließende Bestimmung und Klassifikation derStudienteilnehmer hinsichtlich der Endpunkte.

Eine besondere Problematik für Kohortenstudien mit einer langen Laufzeit ist, dass sich die Klassifikation und Diagnosekriterien von Erkrankungen im Laufe der Zeit ändern können. Beispielsweise hat sich die Definition der TIA geändert [31]. Eine andere Herausforderung sind die Festlegung und Interpretation von Erkrankungszeitpunkten bei schleichend eintretenden Erkrankungen: Der Typ-2-Diabetes ist eine Erkrankung, deren Beginn klinisch meist nicht scharf definiert ist, sondern die schleichend eintritt und häufig erst mit einer Latenzzeit diagnostiziert wird. Darüber hinaus haben sich über die Zeit Diagnosekriterien und -prozeduren geändert, beispielsweise der Stellenwert von $\mathrm{HbAlc}$ und oralem Glukosetoleranztest. Damit in Verbindung steht, dass der Diagnosezeitpunkt inzwischen früher erfolgt; dies muss unter Umständen bei der Interpretation von Zeit-bisEreignis-Daten (Überlebenszeitanalysen) berücksichtigt werden („Lead Time Bias").

\section{Limitationen der Arbeit}

Die Kohortenstudien für den vorliegenden Artikel wurden über die gemeinsame Arbeitsgruppe „Epidemiologie der HerzKreislauf- und Stoffwechsel-Erkrankungen“ der Deutschen Gesellschaft für Epidemiologie (DGEpi), der Deutschen Gesellschaft für Medizinische Informatik, Biometrie und Epidemiologie (GMDS) und der Deutschen Gesellschaft für Sozialmedizin und Prävention (DGSMP) identifiziert. Möglicherweise gibt es darüber hinaus in Deutschland weitere populationsbasierte Kohortenstudien mit bereits erreichten inzidenten kardiovaskulären und metabolischen Erkrankungen, die wir auf diese Weise nicht identifizieren konnten.

\section{Fazit}

Ziel war es, einen Überblick über die Erfassung kardiovaskulärer und metabolischer Endpunkte in populationsbasierten Kohortenstudien in Deutschland zu geben. Dabei wurde deutlich, dass die herangezogenen Datenquellen zwar ähnlich sind, sich aber die Vorgehensweise je nach Endpunkt und Studie unterscheidet. Bei der Identifizierung der Endpunkte spielt die Selbstauskunft von Studienteilenehmern die wichtigste Rolle. Es ist $\mathrm{zu}$ erwarten, dass zukünftig auch andere Datenquellen wie der Abgleich mit Krankenhaus- oder Abrechnungsdaten an Bedeutung zunehmen werden. Von zentraler Bedeutung ist jedoch die Validierung möglicher Endpunkte insbesondere mittels standardisierter Arztbefragungen und Sichtung medizinischer Unterlagen.

Bei vielen Endpunkten wird in den beschriebenen Studien die Sensitivität der Selbstauskunft durch Hinzunahme von weniger spezifischen Informationen erhöht, was wiederum die Notwendigkeit und Bedeutung der Validierung unterstreicht. Unsere Ergebnisse deuten darauf hin, dass eine Kombination verschiedener Herangehensweisen und Datenquellen in Kohortenstudien notwendig ist, um größtmögliche Validität und möglichst vollständige Erfassung aller Ereignisse zu erreichen. Dabei zeigt unsere Übersicht auch, dass diese Prozesse sehr zeit- und personalaufwendig sind, was für die Planung zukünftiger Studien mitbedacht werden sollte.

Darüber hinaus wäre eine allgemeine Standardisierung oder Harmonisierung zu überlegen, um eine Vergleichbarkeit über die Studien hinweg und gemeinsame Analysen zu vereinfachen. Hier gibt es bereits eine enge Zusammenarbeit bestehender Kohortenstudien, um beispielsweise Ergebnisse in unabhängigen Stichproben zu replizieren, Fallzahlen weiter zu erhöhen oder auch geografische Unterschiede zu untersuchen [32-35].

Zusammenfassend bieten die existierenden populationsbasierten Kohortenstudien in Deutschland im Hinblick auf die Identifizierung von inzidenten kardiovaskulären und metabolischen Erkrankungen einen enormen Erfah- rungsschatz, der unter Verwendung der in diesem Artikel zusammengetragenen Informationen in zukünftigen Studien berücksichtig werden kann.

\section{Korrespondenzadresse}

Prof. Dr. T. Pischon, MPH

Forschergruppe Molekulare Epidemiologie, Max-Delbrück-Centrum für Molekulare Medizin in der Helmholtz-Gemeinschaft (MDC) Robert-Rössle-Straße 10, 13125 Berlin, Deutschland

tobias.pischon@mdc-berlin.de

\section{Einhaltung ethischer Richtlinien}

Interessenkonflikt. W.J. Herrmann, C. Weikert, M. Bergmann, H. Boeing, V.A. Katzke, R. Kaaks, D. Tiller, K.H. Greiser, M. Heier, C. Meisinger, C.O. Schmidt, H. Neuhauser, C. Heidemann, C. Jünger, P.S. Wild, S.H. Schramm, K.-H. Jöckel, M. Dörr und T. Pischon geben an, dass kein Interessenkonflikt besteht.

Alle im Artikel beschriebenen identifizierten Studien wurden unter Zustimmung der zuständigen EthikKommission, im Einklang mit nationalem Recht sowie gemäß der Deklaration von Helsinki von 1975 (in der aktuellen, überarbeiteten Fassung) durchgeführt. In allen beschriebenen identifizierten Studien wurden von den Teilnehmenden Einverständniserklärungen zur Studienteilnahme eingeholt.

Open Access. Dieser Artikel wird unter der Creative Commons Namensnennung 4.0 International Lizenz (http://creativecommons.org/licenses/by/4.0/deed. de) veröffentlicht, welche die Nutzung, Vervielfältigung, Bearbeitung, Verbreitung und Wiedergabe in jeglichem Medium und Format erlaubt, sofern Sie den/die ursprünglichen Autor(en) und die Quelle ordnungsgemäßnennen, einen Linkzur Creative Commons Lizenz beifügen und angeben, ob Änderungen vorgenommen wurden.

\section{Literatur}

1. DeStatis (2015) Zahl der Todesfälle im Jahr 2014 um 2,8\% gesunken. Pressemitteilung vom 15 . Dezember 2015 - 465/15. DeStatis, Wiesbaden (www.destatis.de)

2. Plass D, Vos T, Hornberg C, Scheidt-Nave C, Zeeb $H$, Kramer A (2014) Trends in disease burden in Germany: results, implications and limitations of the Global Burden of Disease study. Dtsch Arztebl Int 111:629-638

3. Kolominsky-Rabas PL, Sarti C, Heuschmann PU et al (1998) A prospective community-based study of stroke in Germany - the Erlangen Stroke Project (ESPro): incidence and case fatality at 1,3, and 12 months. Stroke 29:2501-2506

4. Bohley S, Trocchi P, Robra BP, Mau W, Stang A (2015) The regional myocardial infarction registry of Saxony-Anhalt (RHESA) in Germany-rational and study protocol. BMC Cardiovasc Disord 15:45

5. Lowel $H$, Meisinger $C$, Heier $M$, Hormann $A$ (2005) The population-based acute myocardial 
infarction (AMI) registry of the MONICA/KORA study region of Augsburg. Gesundheitswesen 67(Suppl 1):S31-S37

6. Ahrens W, Jockel KH (2015) The benefit of large-scale cohort studies for health research: the example of the German national cohort. Bundesgesundheitsblatt Gesundheitsforschung Gesundheitsschutz 58:813-821

7. Greiser KH, Kluttig A, Schumann B et al (2005) Cardiovascular disease, risk factors and heart rate variability in the elderly general population: design and objectives of the CARdiovascular disease, Living and Ageing in Halle (CARLA) study. BMC Cardiovasc Disord 5:33

8. Wild PS, Zeller T, Beutel M et al (2012) The Gutenberg health study. Bundesgesundheitsblatt Gesundheitsforschung Gesundheitsschutz 55:824-829

9. Boeing H, Wahrendorf J, Becker N (1999) EPICGermany - a source for studies into diet and risk of chronic diseases. European investigation into cancer and nutrition. Ann Nutr Metab 43:195-204

10. Bergmann MM, Bussas U, Boeing H (1999) Followup procedures in EPIC-Germany-data quality aspects. European prospective investigation into cancer and nutrition. Ann Nutr Metab 43:225-234

11. Holle R, Happich M, Lowel H, Wichmann HE, Group MKS (2005) KORA - a research platform for population based health research. Gesundheitswesen 67(Suppl 1):S19-S25

12. Erbel R, Eisele L, Moebus S et al (2012) The Heinz Nixdorf recall study. Bundesgesundheitsblatt Gesundheitsforschung Gesundheitsschutz 55:809-815

13. Volzke H (2012) Study of Health in Pomerania (SHIP). Concept, design and selected results. Bundesgesundheitsblatt Gesundheitsforschung Gesundheitsschutz 55:790-794

14. Gosswald A, Lange M, Kamtsiuris P, Kurth BM (2012) DEGS: German health interview and examination survey for adults. A nationwide cross-sectional and longitudinal study within the framework of health monitoring conducted by the Robert Kochinstitute. Bundesgesundheitsblatt Gesundheitsforschung Gesundheitsschutz 55:775-780

15. National Cohort Consortium G (2014) The German national cohort: aims, study design and organization. Eur JEpidemiol 29:371-382

16. Loeffler M, Engel C, Ahnert P et al (2015) The LIFE-adult-study: objectives and design of a population-based cohort study with 10,000 deeply phenotyped adults in Germany. BMC Public Health 15:691

17. Hamburg City (2016) Hamburg City health study (http://hch-study.com/)

18. Adams HP Jr., Bendixen BH, Kappelle LJ et al (1993) Classification of subtype of acute ischemic stroke. Definitions for use in a multicenter clinical trial. TOAST. Trial of Org 10172 in Acute Stroke Treatment. Stroke 24:35-41

19. The Criteria Committee of the New York Heart Association (1994) Nomenclature and criteria for diagnosis of diseases of the heart and great vessels, 9. Aufl. Little, Brown \& Co, Boston

20. PaulusWJ,TschopeC, Sanderson JEetal(2007) How to diagnose diastolic heart failure: a consensus statement on the diagnosis of heart failure with normal left ventricular ejection fraction by the Heart Failure and Echocardiography Associations of the European Society of Cardiology. Eur Heart J 28:2539-2550

21. Tiller D, Russ M, Greiser KH et al (2013) Prevalence of symptomatic heart failure with reduced and with normal ejection fraction in an elderly general population-the CARLA study. PLoS ONE 8:e59225

22. Hammer GP, du Prel JB, Blettner M (2009) Avoiding bias in observational studies: part 8 in a series of articles on evaluation of scientific publications. Dtsch Ärztebl Int 106:664-668

23. Copeland KT, Checkoway H, McMichael AJ, Holbrook RH (1977) Bias due to misclassification in the estimation of relative risk. Am J Epidemiol 105:488-495

24. Then FS, Luck T, Jacobi F et al (2013) Assessment of mild cognitive impairment and dementia in epidemiologic studies. An overview on the current state of research in Germany. Psychiatr Prax 40:183-191

25. Schelhase T, Weber S (2007) Mortality statistics in Germany. Problems and perspectives. Bundesgesundheitsblatt Gesundheitsforschung Gesundheitsschutz 50:969-976

26. Kircher T, Nelson J, Burdo H (1985) The autopsy as a measure of accuracy of the death certificate. NEngl J Med 313:1263-1269

27. Wolf IK, Knopf $H$, Scheidt-Nave $C$, Kurth BM (2012) Possibilites and limitations of retrospective research on cause of death within the framework of a nationwide epidemiological study. Bundesgesundheitsblatt Gesundheitsforschung Gesundheitsschutz 55:431-435

28. Schmidt CO, Reber K, Baumeister SE, Schminke U Volzke H, Chenot JF (2015) Integration of primary and secondary data in the Study of Health in Pomerania and description of clinical outcomes using stroke as an example. Gesundheitswesen 77:e20-e25

29. Hoffmann W, Bobrowski C, Fendrich K (2008) Secondary data analysis in the field of epidemiology of health care. Potential and limitations. Bundesgesundheitsblatt Gesundheitsforschung Gesundheitsschutz 51:1193-1201

30. Maier B, Behrens S, Busse Ret al (2012) Das Berliner Herzinfarktregister als Beispiel für ein klinisches Register. Public Health Forum 76:18-19

31. Easton JD, Saver JL, Albers GW et al (2009) Definition and evaluation of transient ischemic attack: a scientific statement for healthcare professionals from the American Heart Association/American Stroke Association Stroke Council; Council on Cardiovascular Surgery and Anesthesia; Council on Cardiovascular Radiology and Intervention; Council on Cardiovascular Nursing; and the Interdisciplinary Councilon PeripheralVascular Disease. The American Academy of Neurology affirms the value of this statement as an educational tool for neurologists. Stroke 40:2276-2293

32. Floegel A, Stefan N, Yu Z et al (2013) Identification of serum metabolites associated with risk of type 2 diabetes using a targeted metabolomic approach. Diabetes 62:639-648

33. Hense HW, Koesters E, Wellmann J, Meisinger C, Volzke H, Keil U (2008) Evaluation of a recalibrated systematic coronary risk evaluation cardiovascular risk chart: results from systematic coronary risk evaluation Germany. Eur J Cardiovasc Prev Rehabil 15:409-415

34. StockI D, Ruckert-Eheberg IM, Heier M et al (2016) Regional variability of lifestyle factors and hypertension with prediabetes and newly diagnosed type 2 diabetes mellitus: the population-based KORA-F4 and SHIP-TREND studies in Germany. PLoSONE 11:e156736

35. Neuhauser H (2016) Hypertension in Germany: data from seven population-based studies 1994-2012. Dtsch Ärztebl Int 113(48):809-815. https://doi.org/10.3238/arztebl.2016.0809
36. Schmermund A, Mohlenkamp S, Stang A et al (2002) Assessment of clinically silent atherosclerotic disease and established and novel risk factors for predicting myocardial infarction and cardiac death in healthy middle-aged subjects: rationale and design of the Heinz Nixdorf RECALL Study. Risk Factors, Evaluation of Coronary Calcium and Lifestyle. Am Heart J 144:212-218 